\title{
Definition and realization of the celestial intermediate reference system
}

\author{
N. Capitaine \\ Observatoire de Paris, SYRTE, 61 avenue de l'Observatoire, 75014, Paris, France \\ email: n.capitaine@obspm.fr
}

\begin{abstract}
The transformation between the International Terrestrial Reference System (ITRS) and the Geocentric Celestial Reference system (GCRS) is an essential part of the models to be used when dealing with Earth's rotation or when computing directions of celestial objects in various systems. The 2000 and 2006 IAU resolutions on reference systems have modified the way the Earth orientation is expressed and adopted high accuracy models for expressing the relevant quantities for the transformation from terrestrial to celestial systems. First, the IAU 2000 Resolutions have refined the definition of the astronomical reference systems and transformations between them and adopted the IAU 2000 precession-nutation. Then, the IAU 2006 Resolutions have adopted a new precession model that is consistent with dynamical theories and have addressed definition, terminology or orientation issues relative to reference systems and time scales that needed to be specified after the adoption of the IAU 2000 resolutions. These in particular provide a refined definition of the pole (the Celestial intermediate pole, CIP) and the origin (the Celestial intermediate origin, CIO) on the CIP equator as well as a rigorous definition of sidereal rotation of the Earth. These also allow an accurate realization of the celestial intermediate system linked to the CIP and the CIO that replaces the classical celestial system based on the true equator and equinox of date. This talk explains the changes resulting from the joint IAU 2000/2006 resolutions and reviews the consequences on the concepts, nomenclature, models and conventions in fundamental astronomy that are suitable for modern and future realizations of reference systems. Realization of the celestial intermediate reference system ensuring a micro-arc-second accuracy is detailed.
\end{abstract}

Keywords. standards, astrometry, ephemerides, reference systems, time, Earth

\section{Introduction}

The accurate realization of the celestial and terrestrial reference systems as well as the celestial orientation of the Earth is essential for the reduction of astronomical observations and scientific exploitation. Determining and providing that orientation is coordinated at the international level by the International Service for Earth Rotation and Reference systems (IERS). The IERS products, i.e. the International Terrestrial System (ITRS), the Celestial Reference Systems (ICRS), and the Earth Orientation Parameters (EOP), are based on data provided by the international services (IVS, ILRS, IGS, IDS). Those data are derived from observations by various modern techniques, namely, Very Long Baseline Interferometry (VLBI) of extragalactic radio sources for the IVS, laser ranging of artificial satellites and the Moon for the ILRS, observations with the GNSS systems for the IGS, and observations with the DORIS system for the IDS. Each of these techniques have specific potential for Earth orientation determination.

The transformation between the celestial and terrestrial systems is based on IAU and IUGG standards and models, plus IERS Earth Orientation parameters (EOP). These include (i) polar motion (represented by the $x$ and $y$ coordinates of the direction of the pole in the terrestrial system), which is quasi-periodic and essentially unpredictable, 
(ii) Universal Time, UT1 that provides the variations in the Earth's diurnal angle of rotation and (iii) small adjustments (denoted $\mathrm{d} X$ and $\mathrm{d} Y$ ) to the celestial direction of the pole as predicted by the a priori precession-nutation model.

Recently, IAU and IUGG resolutions have been passed that modify the way the Earth orientation is expressed and adopted the high accuracy models for expressing the EOPs. This has important consequences on the concepts, the nomenclature and the models for fundamental astronomy that are used for modern realization of reference systems.

\section{The IAU 2000/2006 Resolutions on reference systems}

A major change in astronomy was the adoption by the IAU in 1997, of the International Celestial Reference Frame (ICRF) (Ma et al. 1998) that realizes the International Celestial Reference System (ICRS) and, at the same time, of the Hipparcos catalogue, as the realization of the ICRS at optical wavelengths. The transition from the FK5, based on stellar positions and proper motions, to the ICRS, based on observed extragalactic radio-sources, has made it possible to access celestial reference frames at a submilliarcsecond accuracy. Then, several IAU resolutions on reference systems have been passed in 2000 and 2006 that were endorsed by the IUGG in 2003 and 2007, respectively.

\subsection{The IAU 2000 Resolutions}

- IAU 2000 Resolution B1.3 specifies that the systems of space-time coordinates for the solar system and the Earth within the framework of General Relativity are named the Barycentric Celestial Reference System (BCRS) and the Geocentric Celestial Reference System (GCRS), respectively. The corresponding time-coordinates are the Barycentric Coordinate Time (TCB) and the Geocentric Coordinate Time (TCG), respectively. This resolution also provides a general framework for expressing the metric tensor and defining coordinate transformations at the first postNewtonian level (see Soffel et al. 2003) between the BCRS and the GCRS.

- IAU 2000 Resolution B1.5 provides an extended relativistic framework for time transformation in order to give a set of formulas for practical transformations between relativistic time scales (see Soffel et al. 2003).

- IAU 2000 Resolution B1.6 recommends the adoption of the new precessionnutation model that is designated IAU 2000 (version A corresponding to the model of Mathews et al. (2002), of 0.2 mas accuracy and version B corresponding to its shorter version (McCarthy and Luzum 2002) with an accuracy of 1 mas).

- IAU 2000 Resolution B1.7, specifies that the pole of the nominal rotation axis is the Celestial Intermediate Pole (CIP), which is defined as being the intermediate pole, in the ITRS to GCRS transformation, separating its GCRS motion (nutation) from polar motion by a specific convention in the frequency domain.

- IAU 2000 Resolution B1.8 recommends using the "non-rotating origin" (Guinot, 1979), designated Celestial and Terrestrial Ephemeris Origins, as origins on the CIP equator in the celestial and terrestrial reference systems, respectively, and defines UT1 as linearly proportional to the Earth Rotation Angle (ERA) between those origins on the CIP equator (Capitaine et al. 2000). This resolution recommends that the ITRS to GCRS transformation be specified by the positions of the CIP in the GCRS and the ITRS, and the ERA. It also recommends that the IERS continue to provide data and algorithms for the transformations referred to the equinox.

- IAU 2000 Resolution B1.9 provides a re-definition of Terrestrial time (TT) through a conventional linear relation between TT and TCG. 
IAU 2000 Resolutions B1.6, B1.7 and B1.8 came into force on 1 January 2003. At that time, the models, procedures, data and software to implement these resolutions operationally had been made available by the IERS Conventions 2003 and the Standards Of Fundamental Astronomy (SOFA) activities (Wallace 1998). These include the procedure based on non-rotating origins, but also the equinox-based procedure, both being delivered with equal precisions.

\subsection{The IAU 2006 Resolutions}

The precession part in the IAU 2000A model consists only of corrections to the precession rates of the IAU 1976 precession and hence does not correspond to a dynamical theory. This is why IAU 2000 Resolution B1.7 that recommended the IAU 2000A precessionnutation model, recommended at the same time the development of new expressions for precession consistent with dynamical theories and with IAU 2000A nutation. The 2003-2006 IAU Working Group on "Precession and the Ecliptic" (P\&E) looked at several solutions and recommended (Hilton et al. 2006) the adoption of the P03 precession theory (Capitaine et al. 2003); this was endorsed by IAU 2006 Resolution B1. In parallel, the new terminology associated with the IAU 2000 resolutions, along with some additional definitions related to them, were recommended by the 2003-2006 IAU Working Group on "Nomenclature for Fundamental Astronomy" (NFA) (Capitaine et al. 2006) and endorsed by IAU 2006 Resolutions B2 and B3. In summary:

- IAU 2006 Resolution B1 recommends the adoption (from 2009) of the P03 Precession as a replacement to the precession part of the IAU 2000A precessionnutation in order to be consistent with both dynamical theories and the IAU 2000 nutation. It also clarifies the definition of the precession and of the ecliptic.

- IAU 2006 Resolution B2, which is a supplement to the IAU 2000 Resolutions on reference systems, consists of two recommendations:

(1) harmonizing "intermediate" to the pole and the origin (i.e. celestial and terrestrial intermediate origins, CIO and TIO instead of CEO and TEO, respectively) and defining the celestial and terrestrial "intermediate" systems;

(2) fixing the default orientation of the BCRS and GCRS, which is, is unless otherwise stated, assumed to be oriented according to the ICRS axes.

- IAU 2006 Resolution B3 recommends a re-definition of the Barycentric Dynamical Time (TDB) through a conventional linear relation between TDB and TCB.

\section{Consequences of the IAU resolutions on the concepts and definitions}

\subsection{The Barycentric and Geocentric celestial reference systems}

The IAU 2000/2006 Resolutions have provided clear procedures for theoretical and computational developments of the space-time coordinates to be used in the framework of General Relativity. The BCRS, which can be considered to be a global coordinate system for the Solar System, has to be used (with TCB) for planetary ephemerides. In contrast, the GCRS, which can only be considered as a local coordinate system for the Earth, has to be used (with TCG) for Earth rotation, precession-nutation of the equator, motion of Earth's satellite, etc. The spatial orientation of the GCRS is derived from that of the BCRS. Therefore, the GCRS being "dynamically non-rotating", Coriolis terms (that come mainly from geodesic precession) have to be considered when dealing with equations of motion in that system. For all practical applications, unless otherwise stated, the BCRS (and hence GCRS) is assumed to be oriented according to the ICRS axes. The expression of the transformation between the barycentric and geocentric coordinates (i.e. an 
extension of the Lorentz transformation) is defined at the first post-Newtonian level for space coordinates and at the extended level for the time coordinates.

\subsection{The Terrestrial Time and Barycentric Dynamical Time}

The IAU 2000/2006 Resolutions have clarified the definition of the two time scales TT and TDB. TT has been re-defined as a time scale differing from TCG by a constant rate, which is the defining constant. In a very similar way, TDB has been re-defined as a linear transformation of TCB, the coefficients of which are the defining constants. The practical consequences is that TT (or TDB) is for some practical applications more convenient to use than TCG (or TCB) and can be used instead of TCG (or TCB) with the same accuracy. This applies in particular to satellite orbit computations for TT and solar system ephemerides, or analysis of pulsars timings, for TDB.

\subsection{The Celestial Intermediate Pole}

The definition of the CIP has refined the 1980 definition of the Celestial Ephemeris pole in order to realize the pole in the high frequency domain. The CIP has been defined as the intermediate pole, in the transformation from the GCRS to the ITRS, the motion of which is described as follows in the frequency domain. The celestial motion of the CIP includes by convention all the terms with periods greater than 2 days in the GCRS (i.e. frequencies between -0.5 cycles per sidereal day (cpsd) and +0.5 cpsd); the terrestrial motion of the CIP, includes by convention all the terms outside the retrograde diurnal band in the ITRS (i.e. frequencies less than -1.5 cpsd or greater than $-0.5 \mathrm{cpsd}$ ).

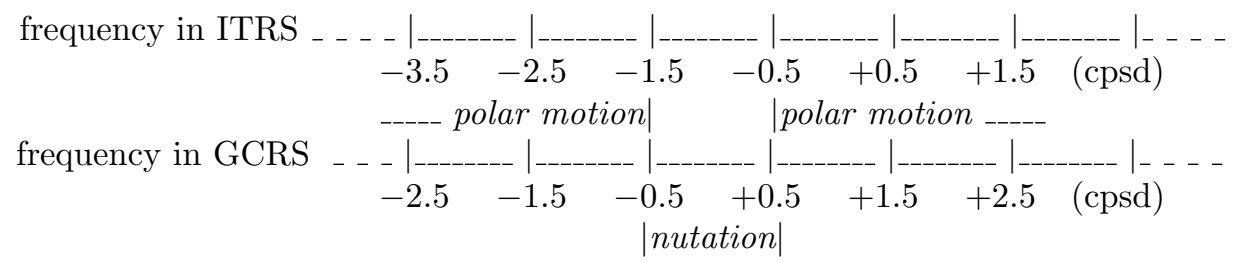

The GCRS position of the CIP replaces the classical precession and nutation quantities (see Fig. 1). The coordinates $X, Y$ of the CIP can be provided by expressions as function of time of the rectangular coordinates, $X=\sin d \cos E ; Y=\sin d \sin E$ of the GCRS direction of the CIP unit vector, which include precession and nutation and the frame bias between the pole of the GCRS and the CIP at J2000.0.

\subsection{The Earth rotation angle}

The diurnal rotation is expressed through the following conventional linear transformation of UT1 (Capitaine et al. 2000), called Earth Rotation Angle (ERA):

$$
\begin{gathered}
\operatorname{ERA}(\mathrm{UT} 1)=2 \pi[0.7790572732640 \\
+1.00273781191135448 \text { (JulianUT1date }-2451545.0)]
\end{gathered}
$$

The linear relationship between ERA and UT1 is a consequence of the kinematically non-rotating nature of the origins to which the ERA refers.

\section{New nomenclature for fundamental astronomy}

The IAU 2000/2006 Resolutions have provided a new paradigm for the GCRS to ITRS transformation, with associated terminology for the pole, the Earth's angle of rotation, the longitude origins and the related reference systems. 

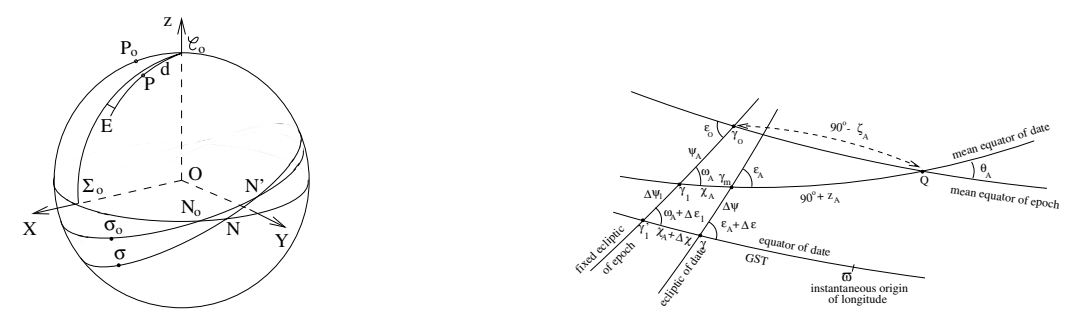

Figure 1. The precession-nutation of the equator with respect to the GCRS: the CIP coordinates (left figure where $\mathrm{P}$ is the CIP) versus the classical precession-nutation angles (right figure)
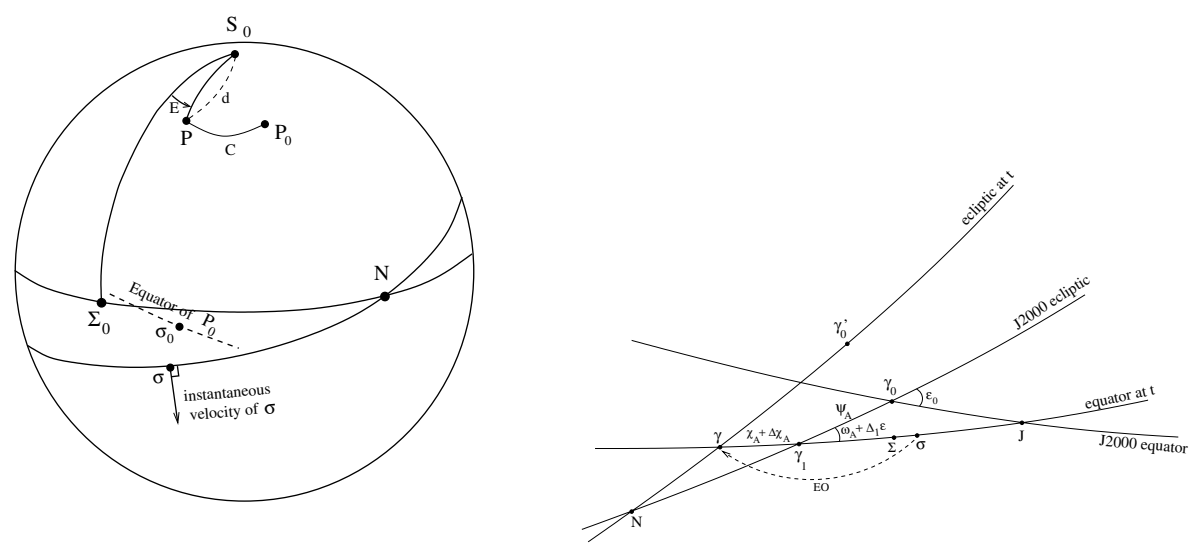

Figure 2. Origin for the Earth angle of rotation with respect to the GCRS: the kinematical definition of the CIO versus the geometrical definition of the classical equinox

\subsection{Terminology choices}

The IAU WG on "Nomenclature for Fundamental Astronomy" (NFA) proposed two IAU 2006 Resolutions, made 14 recommendations, and produced the "IAU 2006 Glossary" including definitions corresponding to the IAU 2000 resolutions as well as new definitions proposed by the WG (including those formally endorsed by the IAU in 2006 or by the IUGG in 2007). In particular, it has been recommended: 1) harmonizing the name of the pole and the origin to "intermediate" and therefore using "Celestial Intermediate Origin" (CIO) and "Terrestrial Intermediate Origin" (TIO), respectively instead of $\mathrm{CEO} / \mathrm{TEO}$; 2) using "equinox based" and "CIO based" for referring to the classical and new paradigms, respectively; 3) using "intermediate" to describe the moving geocentric celestial reference system containing the CIP and the CIO (with its terrestrial counterpart containing the TIO); 4) choosing "equinox right ascension" (or "RA with respect to the equinox") and "intermediate right ascension" (or "CIO right ascension", or "RA with respect to the CIO"), for the azimuthal coordinate along the equator in the classical and new paradigms, respectively; 5) giving the name "equation of the origins" (EO) to the distance between the CIO and the equinox along the intermediate equator, the sign of this quantity being such that it represents the CIO right ascension of the equinox, or equivalently, the difference between the Earth Rotation Angle and Greenwich apparent sidereal time. This has provided a new nomenclature associated with the use of the new origins and also associated with the equatorial coordinates used in an extended way, such that it can be referred to any equator (i.e. GCRS equator or CIRS equator, etc.) and to any origin on those equators (i.e. GCRS origin, CIO, equinox, etc.). 


\subsection{The celestial intermediate reference system}

The celestial intermediate reference system, can be derived from the GCRS by using the GCRS CIP coordinates $X, Y$ and the CIO "locator" (positioning the CIO). Note that the CIO at present is very close to GCRS longitude zero and almost stationary in longitude. The CIO based procedure allows a clear separation between (i) the GCRS motion of the CIP, which is dependent on the precession-nutation model and (ii) the Earth's angle of rotation, ERA, which is not model-dependent. In contrast, precession and nutation are mixed up with Earth's rotation into the expression of Greenwich sidereal time (GST) as function of UT1, which can easily be derived from the following relationship: GST(UT1, TT) = ERA - EO, where EO represents the accumulated precession and nutation in right ascension, directly dependent on the precession-nutation models.

\section{Consequences of the IAU resolutions on high accuracy models}

The IAU 2000/2006 Resolutions have adopted a high precision model for precession and nutation in two successive steps.

The first step was the adoption of the IAU 2000 precession-nutation of Mathews et al. (2002), which has been implemented in the IERS Conventions 2003. This model is composed of a nutation part and a precession part. In addition to the IAU 2000A model are frame bias values between the J2000 mean pole and equinox and the GCRS. The IAU 2000A nutation includes 1365 luni-solar and planetary terms, which are based on rigid Earth nutation transformed with the transfer function based on basic Earth parameters (BEP) that have been fitted to VLBI data. That model is expected to have an accuracy of about $10 \mu$ as for most of its terms. In contrast, the so-called free core nutation (FCN), which is due to geophysical effects and is largely unpredictable, is not part of the model. The precession part, which consists only in corrections to the precession rates of the IAU 1976 precession, was known to not following the predictions of a dynamical theory. The second step in the improvement of the IAU precession-nutation was the adoption (IAU 2006 Resolution B1) of the P03 Precession as a replacement to the precession part of the IAU 2000A precession-nutation in order to be consistent with both dynamical theory and the IAU 2000 nutation. The P03 model has provided improved expressions for both the precession of the ecliptic and the precession of the equator. The latter has taken into account the Earth's $\mathrm{J}_{2}$ rate effect, mostly due to the post-glacial rebound. The P03 precession polynomial developments provide separately the developments for the basic quantities for the ecliptic and the equator that are directly solutions of the dynamical equations, and derived quantities, such as those for the GCRS coordinates of the CIP. These also include expressions for the P03 sidereal time that can been derived form the expression of the Earth Rotation Angle, which is independent of the precession-nutation model and the expression for the equation of the origin, which is directly model-dependent. The various ways of forming the precession-nutation matrix in the new IAU framework have been discussed in Capitaine \& Wallace (2006) and the precession-nutation procedures consistent with IAU 2006 resolutions have been provided in Wallace \& Capitaine (2006).

\section{Conclusions}

The 2000 and 2006 IAU resolutions on reference systems adopted greatly improved numerical models, but also a new paradigm, with associated terminology for the pole, the Earth's angle of rotation, the longitude origins and the related reference systems. 
They have improved the definition and realization of different reference systems and time scales. They have in particular modified the way the Earth orientation is expressed and adopted high accuracy models for expressing the relevant quantities for the transformation from terrestrial to celestial systems. This provides a new framework for dealing with Earth's rotation or computing directions of celestial objects or Earth's satellites in various systems.

A very important effort has been made within the international astronomical community in order to prepare and then to best implement these resolutions, especially through specific IAU Working Groups, the IERS Conventions and the set of software developed by SOFA.

The concepts, nomenclature, models and conventions in fundamental astronomy based on the IAU 2000/2006 Resolutions are suitable for modern and future realizations of the reference systems. This allows the highest accurate realization of the celestial intermediate system linked to the Celestial intermediate pole and origin that replaces the classical celestial system based on the true equator and equinox of date.

The definition and the high accuracy realization of the celestial intermediate reference system based on the IAU 2000/2006 IAU Resolutions is consistent with a micro-arcsecond accuracy. This will allow us to best benefit of the accuracy that is expected to be achieved with future projects related to the celestial reference system, such as Gaia or the VLBI 2010 Observing Programne.

\section{References}

Capitaine, N., Guinot, B., \& McCarthy, D.D., 2000, A\&SA 355, 398

Capitaine, N., Wallace, P. T., \& Chapront, J., 2003, A\&\&A 412, 567

Capitaine, N. \& Wallace, P. T., 2006, A\&A, 450, 855

Capitaine \& the IAU NFA WG, 2007, in Transactions of the IAU XXVIB, van der Hucht, K. A. (ed)

Guinot, B., 1979, in Time and the Earth's Rotation, D. D. McCarthy and J. D. Pilkington (eds), D. Reidel Publishing Company, 7

Hilton, J., Capitaine, N., Chapront, J., et al., Celest. Mech. Dyn. Astr. 94, 3, 351

IAU, 1998, Transactions of the IAU XXIIIB, Anderson, J. (ed), 40

IAU 2000, Transactions of the IAU XXIVB; Manchester, Rickman. H. (ed), Astronomical Society of the Pacific, Provo, USA, 2001, pp. 34-58

IAU 2006, Transactions of the IAU XXVIB; van der Hucht, K. A. (ed)

IUGG 2007, IUGG Resolutions, http://www.iugg.org/resolutions/perugia07.pdf .

IERS Conventions (2003), IERS Technical Note 32, D. D. McCarthy and G. Petit (eds), Frankfurt am Main: Verlag des desamts für Kartographie und Geodäsie, 2004

Ma, C., Arias, E. F., Eubanks et al., 1998, A\& A 116, 516

Mathews, P. M., Herring, T. A. \& Buffett B. A., 2002,J. Geophys. Res. 107, B4, 10.1029/ 2001JB000390

McCarthy, D. D., Luzum, B. J., 2003, Celest. Mech. Dyn. Astr. 85, 37

Soffel, M., Klioner, S. A., Petit et al., 2003, AJ 126, 6, 2687

Wallace P. T., 1998, in Highlights of Astronomy Vol. 11A, J. Andersen (ed.), Kluwer Academic Publishers, 11, 191

Wallace P. T., Capitaine, N., 2006, A\&A 459, 3, 981 